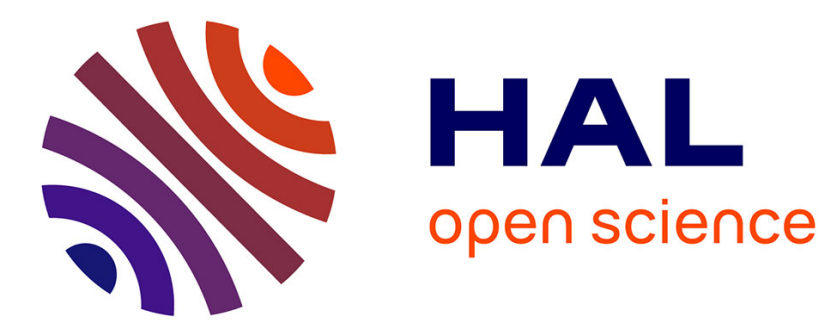

\title{
Hyperquaternion Conformal Groups
}

Patrick Ralph Patrick Girard, Patrick Clarysse, Romaric Pujol, Robert

Goutte, Philippe Delachartre

\section{To cite this version:}

Patrick Ralph Patrick Girard, Patrick Clarysse, Romaric Pujol, Robert Goutte, Philippe Delachartre. Hyperquaternion Conformal Groups. Advances in Applied Clifford Algebras, 2021, 31 (3), 10.1007/s00006-021-01159-y . hal-03270493

\section{HAL Id: hal-03270493 \\ https://hal.science/hal-03270493}

Submitted on 24 Jun 2021

HAL is a multi-disciplinary open access archive for the deposit and dissemination of scientific research documents, whether they are published or not. The documents may come from teaching and research institutions in France or abroad, or from public or private research centers.
L'archive ouverte pluridisciplinaire HAL, est destinée au dépôt et à la diffusion de documents scientifiques de niveau recherche, publiés ou non, émanant des établissements d'enseignement et de recherche français ou étrangers, des laboratoires publics ou privés. 


\section{Advances in Applied Clifford Algebras Hyperquaternion Conformal Groups \\ --Manuscript Draft--}

\begin{tabular}{|c|c|}
\hline Manuscript Number: & AACA-D-21-00031R2 \\
\hline Full Title: & Hyperquaternion Conformal Groups \\
\hline Article Type: & T.C. : ENGAGE 2020 Geometric Algebra for Computing, Graphics \& Engineering \\
\hline Keywords: & Quaternions; Hyperquaternions; Conformal Groups; Canonical Decomposition \\
\hline Corresponding Author: & $\begin{array}{l}\text { Patrick Ralph Girard, Ph.D. } \\
\text { Universite de Lyon } \\
\text { FRANCE }\end{array}$ \\
\hline \multicolumn{2}{|l|}{$\begin{array}{l}\text { Corresponding Author Secondary } \\
\text { Information: }\end{array}$} \\
\hline Corresponding Author's Institution: & Universite de Lyon \\
\hline \multicolumn{2}{|l|}{$\begin{array}{l}\text { Corresponding Author's Secondary } \\
\text { Institution: }\end{array}$} \\
\hline First Author: & Patrick Ralph Girard, Ph.D. \\
\hline \multicolumn{2}{|l|}{ First Author Secondary Information: } \\
\hline \multirow[t]{5}{*}{ Order of Authors: } & Patrick Ralph Girard, Ph.D. \\
\hline & Patrick Clarysse \\
\hline & Romaric Pujol \\
\hline & Robert Goutte \\
\hline & Philippe Delachartre \\
\hline \multicolumn{2}{|c|}{ Order of Authors Secondary Information: } \\
\hline \multicolumn{2}{|l|}{ Funding Information: } \\
\hline Abstract: & $\begin{array}{l}\text { The paper gives a new representation of conformal groups in } n \text { dimensions in terms of } \\
\text { hyperquaternions defined as tensor products of quaternion algebras (or a subalgebra } \\
\text { thereof). Being Clifford algebras, hyperquaternions provide a good representation of } \\
\text { pseudo-orthogonal groups such as } \mathrm{O}(\mathrm{p}+1, \mathrm{q}+1) \text { isomorphic to the } \mathrm{nD} \text { conformal group } \\
\text { with } n=p+q \text {. The representation yields simple expressions of the generators, } \\
\text { independently of matrices or operators. The canonical decomposition and the } \\
\text { invariants are discussed. As application, the } 4 \mathrm{D} \text { relativistic conformal group is detailed } \\
\text { together with a worked example. Finally, the formalism is compared to the operator } \\
\text { representation. Potential uses include in particular, conformal geometry, computer } \\
\text { graphics and conformal field theory }\end{array}$ \\
\hline Response to Reviewers: & All remarks have been taken into account. \\
\hline
\end{tabular}




\title{
Hyperquaternion Conformal Groups
}

\author{
Patrick R. Girard, Patrick Clarysse, Romaric Pujol, Robert \\ Goutte and Philippe Delachartre
}

\begin{abstract}
The paper gives a new representation of conformal groups in $n$ dimensions in terms of hyperquaternions defined as tensor products of quaternion algebras (or a subalgebra thereof). Being Clifford algebras, hyperquaternions provide a good representation of pseudo-orthogonal groups such as $O(p+1, q+1)$ isomorphic to the $n D$ conformal group with $n=p+q$. The representation yields simple expressions of the generators, independently of matrices or operators. The canonical decomposition and the invariants are discussed. As application, the $4 D$ relativistic conformal group is detailed together with a worked example. Finally, the formalism is compared to the operator representation. Potential uses include in particular, conformal geometry, computer graphics and conformal field theory.
\end{abstract}

Mathematics Subject Classification (2010). Primary 15A66; Secondary 11E88, 15A67.

Keywords. Quaternions, Hyperquaternions, Conformal groups, Canonical decomposition.

\section{Introduction}

The $4 D$ conformal group which contains the Poincaré group, is the group of transformations $x \rightarrow x^{\prime}$ of the Minkowski space $E_{1,3}$ (with signature $+---)$ such that $d s^{\prime 2}=\lambda^{2} d s^{2}$ where $\lambda$ is a real number and $d s^{2}=$ $c^{2} d t^{2}-d x^{2}-d y^{2}-d z^{2}$ the metric. This group plays a major role in physics in particular in electromagnetism, general relativity and conformal field theories $[4,18]$. Maxwell's equations (in vacuum) are covariant with respect to the $4 D$ conformal group and in general relativity, twistors constitute an algebraic representation of it $[21,25]$. Generalizing to the $n D$ case (with an arbitrary signature), we shall call them conformal groups $\operatorname{Conf}_{n}(p, q)$ with $n=p+q$. Due to the isomorphism $\operatorname{Conf}_{n}(p, q) \simeq O(p+1, q+1)$, the conformal groups can be expressed as orthogonal groups and represented in particular by Clifford algebras $C_{n+2}(p+1, q+1)$ [2]. Various representations (in 
specific dimensions or signatures) exist and are often formulated in terms of matrices $[15,16,22,24]$. Though we shall focus on the algebraic properties of the conformal groups, geometric aspects have been developed in particular by Hestenes, the Lasenbys, Dorst, Dechant and Hitzer. Adopting the general method presented in [2], we shall introduce a new algebraic representation in terms of hyperquaternions. Historically, hyperquaternions are rooted in the works of Clifford, Lipschitz and Moore [6,17,19,20]. Clifford, applying Grassmann's ideas, introduced his algebras as a tensor product of quaternions and gave a proof thereof. Lipschitz established the formula of $n D$ Euclidean rotations and thereby rediscovered, independently of Clifford, the (even) Clifford algebra (composed of products of an even number of generators). Moore was to call Lipschitz's algebras "hyperquaternions" and gave a canonical decomposition of Euclidean rotations which has been extended to pseudo-Euclidean rotations by the authors [9]. In recent papers, we have applied hyperquaternions to express the unitary, unitary symplectic groups and the Poincaré groups with dual hyperquaternions $[7,9,10]$. Though dual hyperquaternions provide a correct representation of the Poincaré groups, the introduction of a dual element (squaring to zero) might appear as somewhat unnatural. Here, by adding two dimensions to the initial space, we shall introduce such elements within the hyperquaternion framework thereby extending the Poincaré groups to the $n D$ conformal groups. Concerning the isomorphism of hyperquaternions with standard Clifford algebras, a modern proof has been given in [10]. Hyperquaternions yield an intrinsically defined tensor product (without generators), a unique multivector structure (after a generator choice) as well as simple expressions of the generators. The result is an efficient calculus with a straightforward implementation. Hyperquaternions might also open new perspectives of unification. After a few preliminaries, we present the general framework of $n D$ conformal groups. Then, we discuss the canonical decomposition and the invariants. As example, the $4 D$ conformal group is examined and a worked example is provided. Finally, the hyperquaternionic approach is compared to an operator representation. The Lie algebra of the $n D$ conformal group and the explicit multivector structure of the $4 D$ case are given respectively in Appendix $A$ and $B$. Potential applications include in particular, conformal geometry, computer graphics and conformal field theory.

\section{Quaternions, Hyperquaternions and Multivectors}

Quaternions, constituting the quaternion algebra $(\mathbb{H})$, are a set of four real numbers $[7,9,10,12,13,23]$

$$
a=a_{1}+a_{2} i+a_{3} j+a_{4} k
$$

where the elements $i, j, k$ satisfy the fundamental formula

$$
i^{2}=j^{2}=k^{2}=i j k=-1 \text {. }
$$

The quaternion conjugate is $a_{c}=a_{1}-a_{2} i-a_{3} j-a_{4} k$ with $(a b)_{c}=b_{c} a_{c}$. 
Hyperquaternions are defined as tensor products of quaternions $\mathbb{H}, \mathbb{H} \otimes$ $\mathbb{H}, \mathbb{H} \otimes \mathbb{H} \otimes \cdots \otimes \mathbb{H}$ and their subalgebras $\mathbb{C}, \mathbb{H} \otimes \mathbb{C}, \mathbb{H} \otimes \mathbb{H} \otimes \mathbb{C}$, etc.. The tensor product is uniquely defined via formulas such as

$$
\begin{aligned}
& (i, j, k) \otimes 1 \otimes 1=(i, j, k), \\
& 1 \otimes(i, j, k) \otimes 1=(I, J, K), \\
& 1 \otimes 1 \otimes(i, j, k)=(l, m, n)
\end{aligned}
$$

where $(i, j, k),(I, J, K),(l, m, n)$ are distinct commuting quaternionic systems. We shall use the algebra $\mathbb{H} \otimes \mathbb{H} \otimes \mathbb{H}$ in the $4 D$ example. Hyperquaternions yield all real, complex and quaternionic square matrices via the isomorphism $\mathbb{H} \otimes \mathbb{H} \simeq m(4, \mathbb{R})$ where $m(4, \mathbb{R})$ stands for real square matrices of degree 4 .

Hyperquaternions constitute Clifford algebras $C_{n}(p, q)$, over real numbers, having $n=p+q$ generators $e_{i}$ such that $e_{i} e_{j}+e_{j} e_{i}=0(i \neq j), e_{i}^{2}=+1$ ( $p$ generators) and $e_{i}^{2}=-1$ ( $q$ generators) where the generators have a simple compact form (for example $e_{1}=k J, e_{2}=k K l$, etc.). Products of generators yield multivectors $\left(V_{k}\right)(0 \leq k \leq n)$, such as scalars $\left(V_{0}\right)$, vectors $e_{i}\left(V_{1}\right)$, bivectors $e_{i} e_{j}(i<j)\left(V_{2}\right)$, trivectors $e_{i} e_{j} e_{k}(i<j<k)\left(V_{3}\right)$ etc.. The products of an even number of $e_{i}$ constitute the subalgebra $C^{+}$, the rest of the algebra is $C^{-}$. The commutator of two general elements $A, B$ is

$$
[A, B]=(A B-B A) / 2 .
$$

The conjugate $A_{c}$ of a general element $A$ is obtained by replacing the $e_{i}$ by their opposite $-e_{i}$ and reversing the order of the elements

$$
\left(A_{c}\right)_{c}=A, \quad(A B)_{c}=\left(B_{c}\right)\left(A_{c}\right) .
$$

The interior and exterior products of two vectors $a, b$ are given by

$$
2 a \cdot b=(a b+b a), \quad 2 a \wedge b=(a b-b a) .
$$

More generally, for products between a vector and a multivector $A_{p}=a_{1} \wedge$ $a_{2} \wedge \cdots \wedge a_{p}(2 \leq p<n)$, one has

$$
\begin{aligned}
2 a \cdot A_{p} & =\left[a A_{p}-(-1)^{p} A_{p} a\right], \\
2 a \wedge A_{p} & =\left[a A_{p}+(-1)^{p} A_{p} a\right] .
\end{aligned}
$$

Products between multivectors are defined by [5]

$$
\begin{aligned}
A_{p} \wedge B_{q} & =a_{1} \wedge\left(a_{2} \wedge \ldots \wedge a_{p} \wedge B_{q}\right), \\
A_{p} \cdot B_{q} & =\left(a_{1} \wedge \ldots \wedge a_{p-1}\right) \cdot\left(a_{p} \cdot B_{q}\right) \\
& =(-1)^{p(q+1)} B_{q} \cdot A_{p} \quad(p \leq q) .
\end{aligned}
$$

For bivectors one has, in particular

$$
B_{1} B_{2}=B_{1} \cdot B_{2}+B_{1} \wedge B_{2}+\left[B_{1}, B_{2}\right]
$$

with

$$
B_{1} \wedge B_{2}=V_{4}\left[\frac{1}{2}\left(B_{1} B_{2}+B_{2} B_{1}\right)\right] \text {. }
$$




\section{Conformal Groups in $n$ Dimensions}

We develop here the hyperquaternion conformal groups in $n$ dimensions. The method consists in embedding the $n D$ space in an $(n+2) D$ affine space according to the method presented in [2]. We start with the general framework, apply it to the restricted conformal group and then discuss the canonical decomposition as an extension of Moore's method and the invariants [9].

\subsection{General Framework}

To express the conformal group, consider the real hyperquaternion algebra $C_{n}(p, q)$ having $n=p+q$ generators $e_{i}$, a vector space $\left(E_{n}, x=\sum_{i=1}^{n} x_{i} e_{i}\right)$ and the metric

$$
d s^{2}=d x^{2}=\left(d x_{1}^{2}+\cdots+d x_{p}^{2}\right)-\left(d x_{p+1}^{2}+\cdots+d x_{p+q}^{2}\right) .
$$

Vectors are said to be timelike, spacelike and isotropic if respectively $x^{2}$ is positive, negative or nil. The conformal group of $E_{n}$ is defined as the group of transformations $x \rightarrow x^{\prime}$ such that

$$
d s^{\prime 2}=\lambda(x)^{2} d s^{2} \quad(\lambda(x) \in \mathbb{R}) .
$$

To obtain the latter, one embeds the algebra $C_{n}(p, q)$ in the algebra $C_{n+2}(p+$ $1, q+1)$ with two additional generators $e_{0}, e_{n+1}\left(e_{0}^{2}=1, e_{n+1}^{2}=-1\right)$. An affine space is introduced by

$$
\begin{aligned}
X & =\left(\frac{x^{2}-1}{2}\right) e_{0}+x+\left(\frac{x^{2}+1}{2}\right) e_{n+1} \\
& =x^{2} \varepsilon_{1}+x+\varepsilon_{2}
\end{aligned}
$$

with $X^{2}=0$ and

$$
\varepsilon_{1}=\frac{e_{0}+e_{n+1}}{2}, \quad \varepsilon_{2}=\frac{e_{n+1}-e_{0}}{2}, \quad \varepsilon_{1} \wedge \varepsilon_{2}=\frac{e_{0} e_{n+1}}{2}, \quad \varepsilon_{1}^{2}=\varepsilon_{2}^{2}=0 .
$$

The $n D$ conformal group of $E_{n}$ is isomorphic to the orthogonal group $O(p+$ $1, q+1)[2]$. The latter can be constructed from orthogonal symmetries with respect to a plane (going through the origin), perpendicular to a unit vector $u$ (time or spacelike) and given by

$$
X^{\prime}=-\frac{u X u}{u^{2}} .
$$

By combining these symmetries, one obtains the formulas

$$
X^{\prime}= \pm a X a_{c} \quad\left(a a_{c}= \pm 1\right)
$$

where $a \in C_{n+2}^{+}(p+1, q+1)$ or $C_{n+2}^{-}(p+1, q+1)$. The $n D$ conformal group contains the Poincaré group, transversions (also called special conformal transformations) and dilations. The number of parameters equals $\frac{(n+2)(n+1)}{2}$; its Lie algebra is given in Appendix $A$.

If $p$ and $q$ are odd, the conformal group, like the Poincaré group, has four connected components. In all other cases, it has only two connected components [2, p. 88]. An important physical example of the first type is the 
TABLE 1. Hyperquaternion conformal group (R: rotation, $\mathrm{T}$ : translation, $\mathrm{V}$ : transversion, $\mathrm{D}$ : dilation; $m(x)=(1+$ $K x)(1+x K))$

\begin{tabular}{l|l|l|l|l} 
& $R$ & $T$ & $V$ & $D$ \\
\hline$a$ & $e^{e_{i} e_{j} \frac{m^{i j}}{2}}$ & $e^{\varepsilon_{1} P}$ & $e^{\varepsilon_{2} K}$ & $e^{e_{0} e_{n+1} \frac{\varphi}{2}}$ \\
\hline$X^{\prime}\left(=a X a_{c}\right)$ & $x^{2} \varepsilon_{1}$ & $x^{2} \varepsilon_{1}$ & $x^{2} \varepsilon_{1}+x^{\prime}$ & $x^{2} \varepsilon_{1} e^{-\varphi}$ \\
& $+x^{\prime}+\varepsilon_{2}$ & $+x^{\prime}+\varepsilon_{2}$ & $+\varepsilon_{2} m$ & $+x^{\prime}+\varepsilon_{2} e^{\varphi}$ \\
\hline$x^{\prime}$ & $a x a_{c}$ & $(x+P)$ & $x+K x^{2}$ & $x$ \\
\hline$\alpha(x)$ & 1 & 1 & $m(x)$ & $e^{\varphi}$ \\
\hline$y(x)=\frac{x^{\prime}}{\alpha(x)}$ & $a x a_{c}$ & $(x+P)$ & $\frac{x+K x^{2}}{m(x)}$ & $x e^{-\varphi}$
\end{tabular}

TABLE 2. Multiplication rules

\begin{tabular}{c|c|c|c} 
& $\varepsilon_{1}$ & $\varepsilon_{2}$ & $e_{0} e_{n+1}$ \\
\hline$\varepsilon_{1}$ & 0 & $\frac{\left(e_{0} e_{n+1}-1\right)}{2}$ & $\varepsilon_{1}$ \\
\hline$\varepsilon_{2}$ & $-\frac{\left(e_{0} e_{n+1}+1\right)}{2}$ & 0 & $-\varepsilon_{2}$ \\
\hline$e_{0} e_{n+1}$ & $-\varepsilon_{1}$ & $\varepsilon_{2}$ & 1
\end{tabular}

relativistic space-time $(p=1, q=3)$, which we shall develop below. Since all other connected components can be derived from the restricted conformal group Conf $f_{+}^{\uparrow}$ (orthochronous and of determinant 1) we shall now focus on the latter below.

\subsection{Restricted Conformal Group}

The restricted conformal group is defined as

$$
X^{\prime}=a X a_{c}=X^{\prime 0} e_{0}+x^{\prime}+X^{\prime n+1} e_{n+1}
$$

with $a a_{c}=1, a \in C_{n+2}^{+}(p+1, q+1)$. Writing $X^{\prime}=\alpha(x) Y$ with

$$
Y=\left(\frac{y^{2}-1}{2}\right) e_{0}+y(x)+\left(\frac{y^{2}+1}{2}\right) e_{n+1}
$$

where $Y^{2}=0$, one finds $\alpha(x)=X^{\prime n+1}-X^{\prime 0}$ and obtains the expression of the conformal transform of $x$ [2]

$$
y(x)=\frac{x^{\prime}}{\alpha(x)}=\frac{x^{\prime}}{X^{\prime n+1}-X^{\prime 0}} .
$$

Explicitly, the conformal group is constituted by (pseudo-Euclidean) rotations, translations, transversions and dilations as indicated in Table 1 where $X^{\prime}$ is obtained via the multiplication rules given in Table 2.

To verify that the above transformations are indeed conformal ones, one might proceed as follows. Assuming $a$ to be constant (independent of $x$ ), one 
has

$$
d X^{\prime}=a d X a_{c}=d \alpha(x) Y+\alpha(x) d Y
$$

Hence,

$$
d X^{\prime 2}=d X^{2}=d x^{2}=d s^{2}=\alpha(x)^{2} d Y^{2}=\alpha(x)^{2} d y^{2}
$$

where we have used $Y^{2}=0$ (and thus $Y d Y+d Y Y=0$ ). Finally, one finds

$$
d s^{\prime 2} \equiv d y^{2}=\frac{d s^{2}}{\alpha(x)^{2}}=\lambda(x)^{2} d s^{2}
$$

which concludes the verification. The conservation of angles results from

$$
\left[d\left(u^{\prime}+v^{\prime}\right)\right]^{2}=\lambda(x)^{2}[d(u+v)]^{2}
$$

yielding

$$
d u^{\prime} \cdot d v^{\prime}=\lambda(x)^{2} d u . d v .
$$

A standard simple form of $n D$ transversions $x \rightarrow y(x)$ is derived within the Clifford algebra $C_{n}(p, q)$ from

$$
\begin{aligned}
y(x) & =\frac{x+K x^{2}}{(1+K x)(1+x K)}=\frac{x^{2}\left(\frac{x}{x^{2}}+K\right)}{\left(\frac{x}{x^{2}}+K\right) x x\left(\frac{x}{x^{2}}+K\right)} \\
& =\frac{x^{-1}+K}{\left(x^{-1}+K\right)^{2}}=\left(x^{-1}+K\right)^{-1}
\end{aligned}
$$

(where $x^{2}$ is a scalar commuting with $K$ ) and giving

$$
[y(x)]^{-1}=x^{-1}+K .
$$

\subsection{Canonical Decomposition}

Here, we shall apply the canonical decomposition of pseudo-Euclidean rotations given in [9].

Considering the restricted group $S O^{+}(p+1, q+1)$ with $n=p+q=2 k$ (or $2 k+1)$, a rotation within that group can be decomposed into a maximum of $k+1$ orthogonal commuting simple planes $B_{i}[9]$

$$
a=e^{\frac{\Phi_{1}}{2} B_{1}} e^{\frac{\Phi_{2}}{2} B_{2}} \cdots e^{\frac{\Phi m}{2} B_{m}}
$$

where a simple plane is defined by $B_{i} \wedge B_{i}=0$ entailing that $B_{i}^{2}$ is a scalar, $B_{i}^{2} \in\{ \pm 1,0\}$. The simple planes $B_{i}$ can be expressed as

$$
B_{i}=M_{i}+\varepsilon_{1} \wedge P_{i}+\varepsilon_{2} \wedge K_{i}+\varepsilon_{1} \wedge \varepsilon_{2} D_{i}
$$

with $M_{i} \in C_{n+2}^{+}(p+1, q+1)$, and $\left(P_{i}, K_{i} \in E_{n}, D_{i} \in \mathbb{R}\right)$. The bivector $M_{i}$ represents the rotation, the vectors $P_{i}, K_{i}$ respectively the translation and transversion, $D_{i}$ the dilation. Expanding the relation $B_{i} \wedge B_{i}=0$, one obtains

$$
\begin{aligned}
0= & M_{i} \wedge M_{i}+2 \varepsilon_{1} \wedge\left(M_{i} \wedge P_{i}\right) \\
& +2 \varepsilon_{2} \wedge\left(M_{i} \wedge K_{i}\right)+2 \varepsilon_{1} \wedge \varepsilon_{2}\left(D_{i} M_{i}-P_{i} \wedge K_{i}\right)
\end{aligned}
$$

and thus

$$
\begin{aligned}
M_{i} \wedge M_{i} & =0, \quad D_{i} M_{i}=P_{i} \wedge K_{i} \\
M_{i} \wedge P_{i} & =M_{i} \wedge K_{i}=0
\end{aligned}
$$


Hence, $M_{i}$ is a simple plane and the vectors $P_{i}, K_{i}$ are coplanar with $M_{i}$ and anticommute with it $\left(M_{i} P_{i}=-P_{i} M_{i}\right)$, and similarly for $K_{i}$. Developing the term $B_{i}^{2}$ yields

$$
B_{i}^{2}=M_{i}^{2}+\frac{D_{i}^{2}}{4}+P_{i} \cdot K_{i} \in\{ \pm 1,0\}
$$

where $P_{i} \cdot K_{i}=\frac{1}{2}\left(P_{i} K_{i}+K_{i} \cdot P_{i}\right)$ is the interior product of the two vectors. For $B_{i}^{2} \in\{-1,1,0\}$ one has respectively

$$
\begin{aligned}
e^{\frac{\Phi_{i}}{2} B_{i}} & =\cos \frac{\Phi_{i}}{2}+B_{i} \sin \frac{\Phi_{i}}{2}, \\
e^{\frac{\Phi_{i}}{2} B_{i}} & =\cosh \frac{\Phi_{i}}{2}+B_{i} \sinh \frac{\Phi_{i}}{2}, \\
e^{\frac{\Phi_{i}}{2} B_{i}} & =1+B_{i} \frac{\Phi_{i}}{2} .
\end{aligned}
$$

Each component $f_{i}=e^{\frac{\Phi_{i}}{2} B_{i}}$ can be decomposed via the Liouville theorem and its extension to pseudo-Euclidean spaces by Haantjes [14] into a rotation $\left(R_{i}\right)$, a translation $\left(T_{i}\right)$, a transversion $\left(V_{i}\right)$ and a dilation $\left(D_{i}\right)$ for example as

$$
f_{i}=R_{i} T_{i} V_{i} D_{i}
$$

leading for the entire transformation $f=f_{1} f_{2} \cdots f_{m}$ to

$$
\begin{aligned}
f & =\left(R_{1} T_{1} V_{1} D_{1}\right)\left(R_{2} T_{2} V_{2} D_{2}\right) \cdots\left(R_{m} T_{m} V_{m} D_{m}\right) \\
& =\left(R_{1} R_{2} \cdots R_{m}\right)\left(T_{1} T_{2} \cdots T_{m}\right)\left(V_{1} V_{2} \cdots V_{m}\right)\left(D_{1} D_{2} \cdots D_{m}\right) \\
& =R T V D
\end{aligned}
$$

where we have used the commutativity of distinct orthogonal simple planes. Hence, one obtains a complete decomposition.

\subsection{Invariants of the Restricted Conformal Group}

The conformal group being isomorphic to $O(p+1, q+1)$, the invariants are those of the latter group. For $S O(2 m)$ or $S O(2 m+1)$, one has a maximum of $m$ independent Casimir operators commuting with the bivector $B$ generating the group [3]. For the restricted conformal group $S O^{+}(p+1, q+1)$, the invariants can be obtained via the canonical decomposition presented in [9]. Writing

$$
B=b_{1} B_{1}+b_{2} B_{2}+\cdots+b_{m} B_{m}
$$

where $B_{m}$ are orthogonal commuting simple planes and $b_{i}$ are scalars, one computes the bivectors

$$
\begin{aligned}
P_{1} & =B, \\
P_{2} & =(B \wedge B) \cdot B, \\
P_{m} & =\underbrace{(B \wedge B \wedge \cdots \wedge B)}_{m \text { factors }} \cdot \underbrace{(B \wedge B \wedge \cdots \wedge B)}_{m-1 \text { factors }}
\end{aligned}
$$

and the scalars

$$
S_{1}=P_{1} \cdot P_{1}, S_{2}=P_{2} \cdot P_{1}, S_{m}=P_{m} \cdot P_{1}
$$


which commute with $B$ and thus constitute $m$ invariants.

\section{Example: $4 D$ Conformal Group}

The $4 D$ conformal group is of great importance in physics $[4,18]$. We first introduce the algebra, followed by a worked example with a canonical decomposition and the construction of the invariants. Finally, the hyperquaternion representation is compared to an operator representation.

\subsection{Algebra}

Consider the hyperquaternion algebra $\mathbb{H} \otimes \mathbb{H} \otimes \mathbb{H}\left[\simeq C_{6}(2,4)\right]$ having six generators

$$
e_{0}=k I, \quad e_{1}=k J, \quad e_{2}=k K l, \quad e_{3}=k K m, \quad e_{4}=k K n, \quad e_{5}=j
$$

and the multivector structure given in Appendix $A$. The $4 D$ vector space $E_{4}=\operatorname{span}\left(e_{1}, \ldots, e_{4}\right)$ has the metric (with $\left.x=\sum_{i=1}^{4} x_{i} e_{i}\right)$

$$
d s^{2}=d x^{2}=d x_{1}^{2}-d x_{2}^{2}-d x_{3}^{2}-d x_{4}^{2} .
$$

The restricted conformal group is expressed by

$$
X^{\prime}=a X a_{c} \quad\left(a a_{c}=1, \quad a \in C_{6}^{+}(2,4)\right)
$$

with $X=X^{0} e_{0}+x+X^{5} e_{5}$ (and similarly for $X^{\prime}$ ). The group contains the following transformations:

- spatial rotations

$$
a=e^{B \frac{\theta}{2}} \quad\left[B^{2}=-1, \quad B \in(l, m, n)\right]
$$

- boosts

$$
a=e^{B \frac{\theta}{2}} \quad\left[B^{2}=1, \quad B \in(I l, I m, I n)\right]
$$

- space-time translations

$$
a=e^{\varepsilon_{1} P}=1+\varepsilon_{1} P \quad\left[P \in E_{4}, \quad \varepsilon_{1}=\frac{k I+j}{2}, \quad \varepsilon_{1}^{2}=0\right]
$$

- transversions

$$
a=e^{\varepsilon_{2} K}=1+\varepsilon_{2} K \quad\left[K \in E_{4}, \quad \varepsilon_{2}=\frac{j-k I}{2}, \quad \varepsilon_{2}^{2}=0\right]
$$

- dilations

$$
a=e^{-i I \frac{\varphi}{2}} \quad(\varphi \in \mathbb{R})
$$

with a total of 15 parameters. A combination of the above transformations leads to the element $f$

$$
X^{\prime}=f X f_{c} \quad\left(f f_{c}=1, \quad f \in C_{6}^{+}(2,4)\right) .
$$

The canonical decomposition of $f$ yields at most three orthogonal commuting simple planes $B_{i}$

$$
f=e^{B_{1} \frac{\Phi_{1}}{2}} e^{B_{2} \frac{\Phi_{2}}{2}} e^{B_{3} \frac{\Phi 3}{2}}, \quad B_{i}^{2} \in\{ \pm 1,0\}
$$


with $B=b_{1} B_{1}+b_{2} B_{2}+b_{3} B_{3}$ the bivector of $f$ and

$$
B_{i}=M_{i}+\varepsilon_{1} P_{i}+\varepsilon_{2} K_{i}+\varepsilon_{1} \wedge \varepsilon_{2} D_{i} .
$$

One thus obtains a maximum number of three invariants.

\subsection{Worked Example}

As worked example, consider a transformation $X^{\prime}=a X a_{c}$ generated by a dilation, followed by a transversion and a rotation such as

$$
\begin{aligned}
a & =e^{n \frac{\theta}{2}} e^{\varepsilon_{2}(k K m)} e^{e_{0} e_{n+1} \frac{\varphi}{2}} \\
& =\sqrt{\frac{2}{3}}(1+n)+\frac{1}{2 \sqrt{6}}[(J-i K)(l-m)]-\frac{1}{\sqrt{6}} i I(1+n)
\end{aligned}
$$

with $\left(\tan \frac{\theta}{2}=1, \tanh \frac{\varphi}{2}=\frac{1}{2}\right)$ and the bivector $B$ generating the transformation

$$
B=n-\frac{1}{2} i I+\frac{1}{4}(J-i K)(l-m) .
$$

Applying the canonical decomposition presented in [9], one finds

$$
a=e^{B_{1} \frac{\Phi_{1}}{2}} e^{B_{2} \frac{\Phi_{2}}{2}}
$$

where $B_{1}, B_{2}$ are two commuting orthogonal simple planes

$$
\begin{aligned}
& B_{1}=\frac{1}{10}(J-i K)(3 l-m), \\
& B_{2}=-\frac{1}{10}(J-i K)(l+3 m)
\end{aligned}
$$

with $B_{1}^{2}=-1, B_{2}^{2}=1\left(\tan \frac{\Phi_{1}}{2}=1, \tanh \frac{\Phi_{2}}{2}=\frac{1}{2}\right)$. To obtain the invariants, one computes, as indicated above, the quantities

$$
\begin{aligned}
& P_{1}=B, \\
& P_{2}=(B \wedge B) \cdot B=\frac{1}{2}+i I+\frac{1}{4}(J-i K)(l+m), \\
& P_{3}=(B \wedge B \wedge B) \cdot(B \wedge B)=0
\end{aligned}
$$

and the scalars

$$
S_{1}=P_{1} \cdot P_{1}=-\frac{3}{4}, \quad S_{2}=P_{2} \cdot P_{1}=-1, \quad S_{3}=P_{3} \cdot P_{1}=0
$$

yielding the two conformal invariants $S_{1}, S_{2}$.

\subsection{Other Representation}

Above, we have given an algebraic representation of the $n D$ conformal groups. Other representations exist in particular in terms of operators with the correspondence $[2$, p. 135]

- rotations: $e_{i} e_{j} \rightarrow x_{i} \frac{\partial}{\partial x_{j}}-x_{j} \frac{\partial}{\partial x_{i}}$,

- translations: $\left(e_{0}+e_{n+1}\right) e_{i} \rightarrow \frac{\partial}{\partial x_{i}}$,

- transversions: $\left(e_{n+1}-e_{0}\right) e_{i} \rightarrow x^{2} \frac{\partial}{\partial x_{i}}-2 x_{i} x^{k} \frac{\partial}{\partial x_{k}}$,

- dilations: $e_{0} e_{n+1} \rightarrow x_{i} \frac{\partial}{\partial x_{i}}$. 
Both representations lead to the same Lie algebra, given in Appendix $A$. Operators are used in quantum mechanics and quantum field theories [1]. Physical applications of conformal groups include in particular electromagnetism, general relativity and conformal field theory $[4,18]$.

\section{Conclusion}

In this paper, we have developed a new algebraic representation of conformal groups in $n$ dimensions in terms of hyperquaternions. The representation is distinct from matrix ones and gives simple expressions of the generators. After the general formalism, the canonical decomposition and the invariants have been discussed. As concrete example, the $4 D$ relativistic case has been detailed together with a worked example. Finally, the representation has been compared to an operator representation. It is hoped that the hyperquaternionic approach might advance the understanding of the algebraic structure of conformal groups, provide an efficient operational calculus and open new unification perspectives. Potential applications include in particular, conformal geometry, computer graphics and conformal field theory.

\section{Acknowledgment}

This work was supported by the LABEX PRIMES (ANR-11-LABX-0063) and was performed within the framework of the LABEX CELYA (ANR10-LABX-0060) of Université de Lyon, within the program "Investissements d'Avenir" (ANR-11-IDEX-0007) operated by the French National Research Agency (ANR). The authors gratefully acknowledge the comments of the reviewers which have greatly improved the readability of the paper.

\section{Appendix A. Lie Algebra of the $n D$-Conformal Group}

Consider an $n D$ space embedded in an $n+2$ hyperquaternion algebra with the generators $e_{0}, e_{1}, \ldots, e_{n}, e_{n+1}$. The Lie generators of the rotations, translations, transversions and dilations of the restricted conformal group are respectively

$$
\begin{aligned}
M_{i j} & =\frac{1}{2} e_{i} e_{j} \quad(1 \leq i, j \leq n, i \neq j) \\
P_{i} & =\left(\frac{e_{0}+e_{n+1}}{2}\right) e_{i}, \quad K_{i}=\left(\frac{e_{n+1}-e_{0}}{2}\right) e_{i} \\
D & =\frac{e_{0} e_{n+1}}{2}
\end{aligned}
$$


One derives easily the following Lie commutators $[A, B]=A B-B A$, with $\eta_{i j}=\left(e_{i} e_{j}+e_{j} e_{i}\right) / 2$.

$$
\begin{aligned}
{\left[M_{i j}, M_{k l}\right] } & =\eta_{j k} M_{i l}+\eta_{i l} M_{j k}-\eta_{j l} M_{i k}-\eta_{i k} M_{j l}, \\
{\left[M_{i j}, P_{k}\right] } & =\eta_{j k} P_{i}-\eta_{i k} P_{j}, \quad\left[M_{i j}, K_{k}\right]=\eta_{j k} K_{i}-\eta_{i k} K_{j} \\
{\left[M_{i j}, D\right] } & =\left[P_{i}, P_{j}\right]=\left[K_{i}, K_{j}\right]=0, \\
{\left[K_{i}, P_{j}\right] } & =2 \eta_{i j} D+M_{i j}, \quad\left[D, P_{i}\right]=-P_{i}, \quad\left[D, K_{i}\right]=K_{i} .
\end{aligned}
$$

\section{Appendix B. Multivector structure of $\mathbb{H} \otimes \mathbb{H} \otimes \mathbb{H}$}

A general hyperquaternion $A$ is a set of 64 terms which can be grouped into a set of 16 quaternions $\left[q_{i}\right]=a_{i}+b_{i} l+c_{i} m+d_{i} n$ with respect to the sets $i j k / I J K / l m n$

$$
\begin{aligned}
A= & {\left[q_{1}\right]+I\left[q_{2}\right]+J\left[q_{3}\right]+K\left[q_{4}\right] } \\
& +i\left[q_{5}\right]+i I\left[q_{6}\right]+i J\left[q_{7}\right]+i K\left[q_{8}\right] \\
& +j\left[q_{9}\right]+j I\left[q_{10}\right]+j J\left[q_{11}\right]+j K\left[q_{12}\right] \\
& +k\left[q_{13}\right]+k I\left[q_{14}\right]+k J\left[q_{15}\right]+k K\left[q_{16}\right]
\end{aligned}
$$

yielding a multiplication table which can be implemented (algebraically or numerically) on Mathematica using its quaternion product $\left[q_{i}\right] * *\left[q_{j}\right]$. The complete multivector structure is given below, where $e_{0123}=e_{0} e_{1} e_{2} e_{3}$, etc..

$$
\left[\begin{array}{llll}
1 & l=e_{34} & m=e_{42} & n=e_{23} \\
I=e_{1234} & I l=e_{21} & I m=e_{31} & I n=e_{41} \\
J=e_{2034} & J l=e_{02} & J m=e_{03} & J n=e_{04} \\
K=e_{10} & K l=e_{1034} & K m=e_{0124} & K n=e_{1023} \\
i=e_{012345} & i l=e_{1025} & i m=e_{1035} & i n=e_{1045} \\
i I=e_{50} & i I l=e_{3045} & i I m=e_{4025} & i I n=e_{2035} \\
i J=e_{51} & i J l=e_{3145} & i J m=e_{4125} & i J n=e_{2135} \\
i K=e_{2345} & i K l=e_{52} & i K m=e_{53} & i K n=e_{54} \\
j=e_{5} & j l=e_{345} & j m=e_{542} & j n=e_{235} \\
j I=e_{12345} & j I l=e_{215} & j I m=e_{531} & j I n=e_{541} \\
j J=e_{32450} & j J l=e_{025} & j J m=e_{035} & j J n=e_{045} \\
j K=e_{105} & j K l=e_{10345} & j K m=e_{01245} & j K n=e_{10235} \\
k=e_{10234} & k l=e_{012} & k m=e_{013} & k n=e_{014} \\
k I=e_{0} & k I l=e_{034} & k I m=e_{204} & k I n=e_{023} \\
k J=e_{1} & k J l=e_{134} & k J m=e_{214} & k J n=e_{123} \\
k K=e_{324} & k K l=e_{2} & k K m=e_{3} & k K n=e_{4}
\end{array}\right]
$$

A Mathematica notebook, concerning $\mathbb{H} \otimes \mathbb{H} \otimes \mathbb{C}$ is provided in [8].

\section{References}

[1] Adler, S.L.: Quaternionic Quantum Mechanics and Quantum Fields. Oxford University Press, New York (1995) 
[2] Anglès, P.: Conformal Groups in Geometry and Spin Structures. Birkhäuser, Boston (2008)

[3] Bincer, A.M.: Lie Groups and Lie Algebras. Oxford University Press, Oxford (2013)

[4] Blumenhagen, R., Plauschinn, E.: Introduction to Conformal Field Theories: With Applications to String Theories. Lect. Notes Phys. 779. Springer, Berlin (2009)

[5] Casanova, G.: L'algèbre vectorielle. PUF, Paris (1976)

[6] Clifford, W.K.: Applications of Grassmann's extensive algebra. Amer. J. Math. 1, 266-276 (1878)

[7] Girard, P.R., Clarysse, P., Pujol, R., Goutte R., Delachartre, P.: Dual Hyperquaternion Poincaré Groups. Adv. Appl. Clifford Algebras 31, 15 (2021). https://doi.org/10.1007/s00006-021-01120-z.

[8] Girard, P.R., Clarysse, P., Pujol, R., Goutte R., Delachartre, P.: DUAL HYPERQUATERNION POINCARE GROUPS.nb. Mathematica notebook. Academic Articles and Supplements. http://www.notebookarchive.org/2021-034 mrwcdg/

[9] Girard, P.R., Clarysse, P., Pujol, R., Goutte, R., Delachartre, P.: Hyperquaternions: An efficient mathematical formalism for geometry. In: Nielsen F., Barbaresco F. (eds), Geometric Science of Information 2019. Lecture Notes in Computer Science 11712, 116-125, Springer, Cham (2019).

[10] Girard, P.R., Clarysse, P., Pujol, R., Goutte, R., Delachartre, P.: Hyperquaternions: A new tool for physics. Adv. Appl. Clifford Algebras 28, 68 (2018). https://doi.org/10.1007/s00006-018-0881-8

[11] Girard, P.R., Clarysse, P., Pujol, R., Wang, L., Delachartre, P.: Differential geometry revisited by biquaternion Clifford algebra. In J.-D. Boissonnat et al. (Eds.): Curves and Surfaces. Springer (2015).

[12] Girard, P.R.: Quaternions, Clifford Algebras and Relativistic Physics. Birkhäuser, Basel (2007)

[13] Girard, P.R.: Algèbre de Clifford et Physique relativiste. PPUR, Lausanne (2004)

[14] Haantjes, J.: Conformal representations of an $n$-dimensional euclidean space with a non-definite fundamental form on itself. Proc. Ned. Akad. Wet. (Math) 40, 700-705 (1937)

[15] Helmstetter, J.: Conformal Groups and Vahlen Matrices. Adv. Appl. Clifford Algebras, 27, 33 (2017)

[16] Lasenby, J. et al.: Calculating the Rotor Between Conformal Objects. Adv. Appl. Clifford Algebras, 29, 102 (2019)

[17] Lipschitz, R.: Principes d'un calcul algébrique qui contient comme espèces particulières le calcul des quantitiés imaginaires et des quaternions. C.R. Acad. Sci. Paris 91, 619-621, 660-664 (1880)

[18] Kastrup, H.A.: On the advancements of conformal transformations and their associated symmetries in geometry and theoretical physics. Ann. Phys (Berlin) 17, 631-690 (2008)

[19] Moore, C.L.E.: Hyperquaternions. J. Math. Phys. 1, 63-77 (1922) 
[20] Moore, C.L.E.: Rotations in hyperspace. In: Proceedings of the American Academy of Arts and Sciences 53, 8, 651-694 (1918)

[21] Penrose, R.: Twistor algebra. J. of Math. Physics, 8 (2), 345 (1967)

[22] Porteous, I.: Clifford Algebras and the Classical Groups. Cambridge University Press, Cambridge (1995)

[23] Vince, J.: Quaternions for Computer Graphics. Springer, London (2011)

[24] Vince, J.: Geometric Algebra for Computer Graphics. Springer, London (2008)

[25] Ward, R.S., Wells, R.O.: Twistor Geometry and Field Theory. Cambridge University Press, Cambridge (1990)

Patrick R. Girard

Univ Lyon, INSA-LYON, Université Claude Bernard Lyon 1, UJM-Saint Etienne, CNRS, Inserm, CREATIS UMR 5220, U1206, F-69621 LYON, France

Bât Blaise Pascal

7 avenue Jean Capelle

F-69621 Villeurbanne, France

e-mail: patrick.girard@creatis.insa-lyon.fr

Patrick Clarysse

Univ Lyon, INSA-LYON, Université Claude Bernard Lyon 1, UJM-Saint Etienne, CNRS, Inserm, CREATIS UMR 5220, U1206, F-69621 LYON, France

Bât Blaise Pascal

7 avenue Jean Capelle

F-69621 Villeurbanne, France

Romaric Pujol

Pôle de Mathématiques, INSA-Lyon

Bât. Léonard de Vinci, 21 avenue Jean Capelle

F-69621 Villeurbanne, France

Robert Goutte

Univ Lyon, INSA-LYON, Université Claude Bernard Lyon 1, UJM-Saint Etienne, CNRS, Inserm, CREATIS UMR 5220, U1206, F-69621 LYON, France

Bât Blaise Pascal

7 avenue Jean Capelle

F-69621 Villeurbanne, France

Philippe Delachartre

Univ Lyon, INSA-LYON, Université Claude Bernard Lyon 1, UJM-Saint Etienne, CNRS, Inserm, CREATIS UMR 5220, U1206, F-69621 LYON, France

Bât Blaise Pascal

7 avenue Jean Capelle

F-69621 Villeurbanne, France 